$\xi_{p-1}=-1$

\title{
Inevitable dissection of left ventricle aimed at discovery of cardiac blood flow appraisal
}

\author{
Inbalatha M. ${ }^{*}$, Kalaivani S. ${ }^{2}$ \\ ${ }^{1}$ Research Scholar, SENSE, VIT, Vellore, Tamil Nadu, India, \\ ${ }^{1}$ Assistant Professor, Department of ECE, Dr.T.T.I.T, KGF, Karnataka, India. \\ ${ }^{2}$ Associate Professor, SENSE, DSP Division, VIT, Vellore, Tamil Nadu, India. \\ *Corresponding author E-mail: inbalathaphd@gmail.com
}

\begin{abstract}
Cardiac Magnetic Resonance Images (MRI) are chastely indispensable implement for truthful demarcation of the left Ventricle myocardium for purposeful and serviceable cardiac examination. Dissection of MRI principally the leftward ventricle (LV) is a blistering focus with extraordinary perplexing owed to deceive delineation such as percentages misalignment in its position, in homogeneity of sequential portions and to clinch divergence in focus of adjoining pixels for the change in the body fluid drift. In this effort, we put forward a scheme to inevitably fragment the leftward Ventricle of MRI images by Round Hough Transform (RHT) initially by locating the seed point stalked a circle with apt radius as basal region and verdict the ROI in the image. Adjoining the center, apex region of the left ventricle is positioned till the extent and shape connection obsolete plus the mid region from the intermediate ventricle to the base up to the myocardial fringe. This same images of 21 subjects are compared with the manual delineation method by locating the center manually. Mean and Standard deviation is calculated for the basal, mid and apex regions of the LV for auto and manual segmentation. Bland -Altman investigation is performed for End Diastolic Measurements in volume (EDV) with the existing investigations and found to be more close to the ground truth results and superior than the previously enduring methods. To conclude the projected segmentation process truthfully locates and segments the LV which trials the one hundredth of blood pour. EDV measurements are established with less computational time to find the patient's heart failure with myocardial Infarction.
\end{abstract}

Objective: To spot the cardiac maladies triggered owed to stumbling block of blood by dissection of left Ventricle with MRI image.

Keywords: Bland-Altman Analysis, Circular Hough Transform, Dissection, End Diastolic Volume, and MRI.

\section{Introduction}

Cardiac MRI skill display the communal heart structural and efficient facts for verdict and cure of cardiovascular diseases by a noninvasive mode [1]. To quantify the left ventricle (LV) volumes, different imaging types like echo cardiograph, Computed Tomography imaging methods in addition to Magnetic Resonance imaging remain in rehearsal, and moreover this imaging procedureconsumestheirreplaceablecapabilitytoaffordbodilyimager ies of the LV with organized extraordinary chronological and altitudinal determinations, devoid of baring the longsuffering to ionizing contamination or divergence causes [2]. CMRI centered volumetric extents have been before hand exposed to be extremely reproducible $[3,4]$ and are habitually cast off the impost of LV role [5] even thru high- intensity isometrics [6] otherwise in heart malady [7]. Splitting up of structural configurations from heart MR imageries, chiefly the left-hand side ventricle, is essentially intended for figuring investigative directories like expulsion fraction, ventricular measurements in volume be sides barrier stiffening [8]. Splitting up of the left ventricle rests on solitary open disputes for the reason of half- volume upshot, stuffs resultant from whirling blood, papillary muscle, uproars and image glitches [9]. A lot of exertions have been keen to elucidate this difficulties together with morphologic dissections [10], fuzzy bundling [11,12], model-based methodologies[13, 14], active contours [15 18], and level set procedures [19]. Blue-collar dissection is tougher and receipts added stint in medical isometrics that clues to improve a new reflex method to abstract LV outlines instinctively. In recent times, diastolic role impost built on LV volume worked efficiently with CMR imaging methods [20, 21]. Old-style LV volume valuation is restricted to the investigation of systolic enactment via labor-intensive delimitation of the left ward ventricle endocardium at both the systolic and diastolic end stages, also consumes only 5-10 minutes to physically demarcate LV endocardium for a hoard of around 20 images of the two stages [22]. Diastolic role calculation is quiet obligatory and entails dissection of the volume through hall progressive segments. This out comes in al of to f200 images necessitating investigation, which is not real for manual delineation.

Contemporary diastolic functional appraisals are accomplished with stead fasts of ware with labor-intensive dissection of basal section in 25 minutes via skilled radiologist [21-23]. Even though there is suggestion to upkeep that the quantifiable productivity for LV exploration concerning the universally hand-me-down software correspondences is not considerably diverse [24], the main change ability of practical dimensions resulting from LV subdivision is powerfully predisposed by the pictorial assortment of the basal slice and blue Collar explanation of LV endocardium [7]. Consequently, entirely programmed subdivision and quantification are compulsory and of prospective medical efficacy for stretched solicitations of CMR in monotonous scientific rehearsal. 


\section{Related works}

Amongst surviving robotic techniques [25], methodologies with solid former awareness, such as active appearance model [26], Gaussian-mixture model [27], arbitrary strides [28,29], and graph cuts[3032], are in effect for mining precise basal LV and rejecting specifics resembling definite and trabecular muscles from LV. Tactics with fragile or no earlier awareness, such level sets[33], Active contour model [34], iterative threshold-decreasing region growth [35] or multi seed region growth [36], and dynamic programming [37], naturally necessitate manual credentials of the basal slices. In count, methodologies using long and short axis images correspondingly requisite labor-intensive involvement [3840] to choice the spots of the apex and mitral valves plus the curve of long axis.

Effusively instinctive LV dissection has been challenging to succeed in rehearsal. The universally cast off cine SSFP acquirement of the heart take account of shares outside the the a pica land basal slices of LV, which stay both physically omitted [35] or unheeded overall[36,37,41]in utmost of the robotic dissection. The basal slice regularly comprises slices of the aortic origin and is for that reason not absolutely fenced by ventricular myocardium, creating it a task to robotically fragment. The circular Hough transform castoff to fragment the leftward ventricle body fluid pool as of short-axis view heart pictures lacking on detailed profile outline familiarity [42]. Bansod utilizes the confined rise route of spitting image pixel intensity to streamline the CHT used for rough calculation of the leftward ventricle blood puddle by considering the two dimensional probe window near the center [43]. Fern'andez smeared HT elliptically towards SA view images, using Gaussian Derivative by means of leveling and outline boundary revealing worker which in future used as a prime input for active contour delineation with LA Segmentation [44].

\subsection{Rounded Hough Transform [RHT]}

The RHT is a feature mining procedure cast off in appearances cru tiny and processor exposure for lines findings in the image,but later enhanced to identify circles and ellipses[45]. Circular Hough Transform also known as RHT approach used for finding circles in images owing to its superior characteristics like its healthy and vigor robustness to noise, change in intensity lighting and any other impediment in the image. The important steps for consideration are accumulator array reckoning, Centre and radius guesstimate. The foreground high intensity bright pixels are the Candidate pixels cast 'votes' in the array and this will be occurring in a sequence around the center to form a circle of fixed radius are plainly valued with the center of the circles based on circular histograms of the acquired image. The voting structure for CHT is shown in Fig.1. The diagram indicates that the candidate pixel will always lie on the circle and the votes of them will be accumulated and coincide to the center of the circle and the radius is appraised. In mat lab, the function in find circles is used to find circles for an image which uses two dealings Phase- coding by evasion and Two-stage [46].The Fig.2 depicts the mode of voting for dissimilar radii towards route of the gradient with different center and its corresponding radius. The steps for the circle detection process are summarized below.
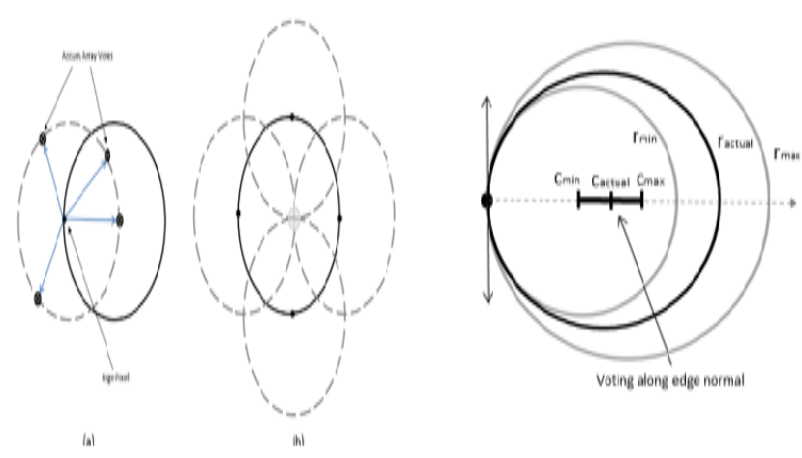

Fig.1: Voting Structure of CHT Fig. 2: Voting with different radius

\subsection{Algorithm Steps}

- Initially build a accumulator matrix for each pixel in the image.

- Consider all the cells pixels to zero at the beginning.

- Consider every edge point $(\mathrm{x}, \mathrm{y})$ directing the image position.

- Increment all cell values using the circle equation $(x-a)^{2}+$ $(y-b)^{2}=r^{2}$ to locate the focus point.

- Each possible values of 'a', corresponding possible values of ' $b$ ' such that it satisfies the above equation.

- Find the local maxima cells in the accumulator matrix which finds circles is calculated by the sum.

- If radius of the circle is not known, a 3D accumulator space $(\mathrm{x}, \mathrm{y}, \mathrm{r})$ is picked such that $\mathrm{x}, \mathrm{y}$ directs the coordinates and $\mathrm{r}$ directs the circle radius.

\section{Methods}

In the proposed method, Cardiac MRI images of 21 subjects of different slices of seven normal and fourteen abnormal LV images are chosen arbitrarily from the Cardiac Atlas Database. The Database used for the work, Sunnybrook Cardiac Data similarly acknowledged by means of the 2009 Cardiac Magnetic Resonance Leftward Ventricle Dissection Experiment facts, comprise of forty five SA MRI imageries as of a diverse of patients of both gender with different age groups and pathologies. In our case, heart failure with infarction patient data and others are taken to find the \% of blood flow and blockage during EDV. The size of the image is resized to $256 * 256$ followed by converting into gray scale image to reduce the complexity and storage area. An automated LV segmentation of MRI image starts with identifying the circular shaped region from the image. Locating the seed point or center is the primary task which is equipped by Circular Hough Transform that stores the each cell values in the accumulator. The values more than zero positions for circle edge with high intensity and values less than zero positions for dark circles with less intensity pixel values [42]. It is observed from the LV image, the bright region which reflects radiation in the myocardium refers to blood pool and the black region which absorbs radiations refers to blockage. Two concentric circles will be marked tarnished for the inner and outer layer. Adjacent to the center, Apex point will be located, by finding the area of the inside circle. Let the high intensity pixels be $\mathrm{Y}$ to represent blood flow and dark intensity pixels be $\mathrm{X}$ to represent blockage. Then the area in pixels will be (Y/X).

If $\left\{\begin{array}{r}\mathrm{X}=\mathrm{Y}, \text { No blockage } \\ \mathrm{Y}<\mathrm{X} \text {, blockage }\end{array}\right.$

Then keeping the seed point as reference, the CHT draws a circle with a radius of $30 \mathrm{~mm}$ which is the basal region and this will be ROI by eliminating the other information of the image. Three regions of the myocardial LV like apex, mid and Basal region are clearly executed step by step to explore the blood flow in 
penetration till the tip point of the left-hand side ventricle of the core. When the basal region is detected, then the blood flow region with white high intensity pixels will be marked as the mid region. Then immediately morphological operation erosion will be done in the detected mid region to detect the apex. The apex will be probably more close to the seed point where there may be blood flow or blockage with dark pixels. The complete stream diagram of the segmentation of left Ventricle using automated RHT and manual segmentation is shown in Fig. 3 .

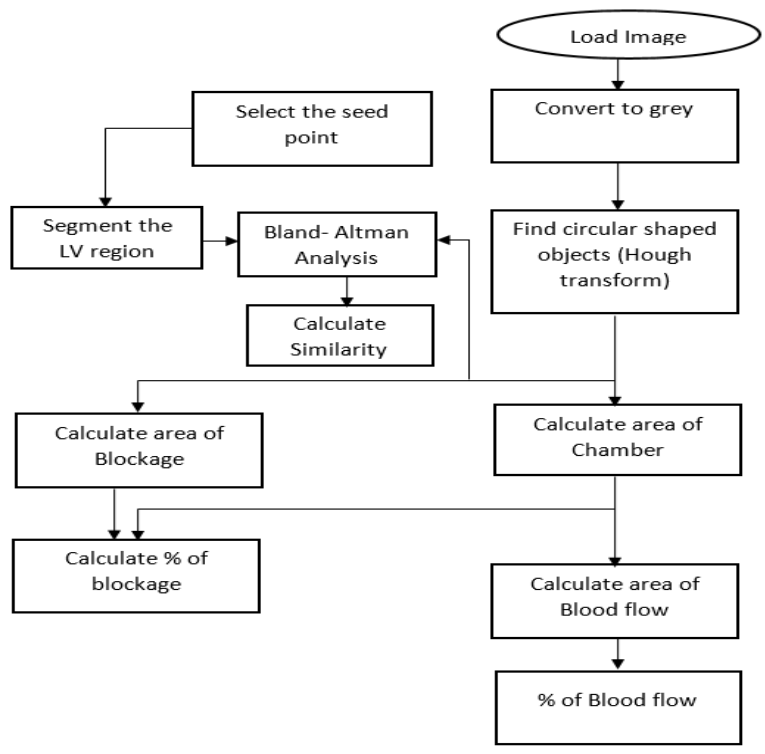

Fig.3: Stream Diagram for the LV Segmentation using CHT and manual

The same images were manually forced to select the seed point and segment the left ventricle. Calculate the area of the chamber, area of the blockage which clues to volume of blood flowin the LV during the End Diastolic. Bland -Altman Analysis is performed to find the correlation or similarity with the proposed method and manual method followed by the existing methods [**] to find the accuracy of the automatic segmentation using Circular Hough Transform. Mean and Standard Deviation are calculated for automated and manual for three different regions. After segmenting the three regions, $\%$ of blood flow is be calculated with the ratio of total pixels of mid region and basal region of the left ventricle and also the $\%$ of blockage is calculated.

\section{Results and discussion}

The proposed Round Hough Transform is implemented on the input MRI image, which first locates the seed point indicated by red color, then the maximum area of basal region will be the ventricular myocardium specified in red circle besides the mid region designated in green color stands for the total span of the papillary muscles and the tip region of the image apex region with the blue color extending the papillary muscle towards the apex. The apex region as discussed could have or not have flow of blood. The blood region is marked with yellow color with high intensity in the LV. The results for four subjects are shown in the Fig. 4 with the automatically segmented three regions of the LV and the blood flow region.
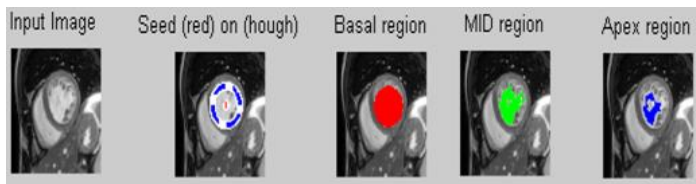

Blood region (edge)
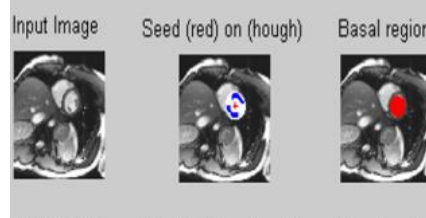

MID region

Apex region Blood region (edge
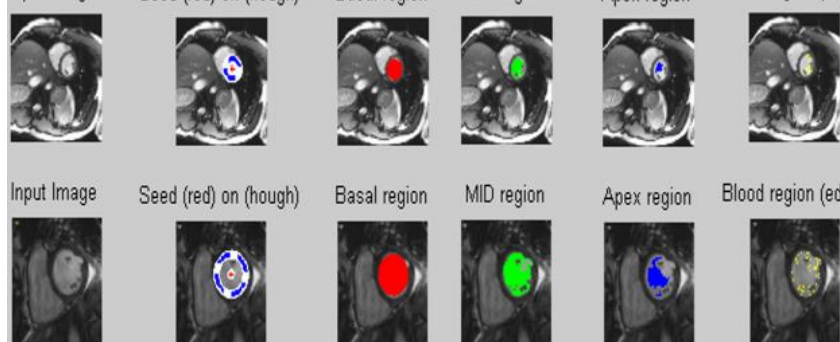

Seed (red) on (hough)

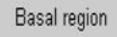

MID region

Apex region Blood region (edge
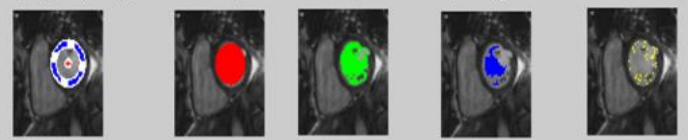

Input Image
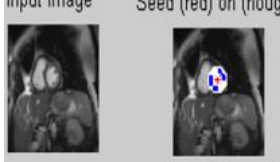

Basal region

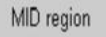

Apex region Blood region (edge
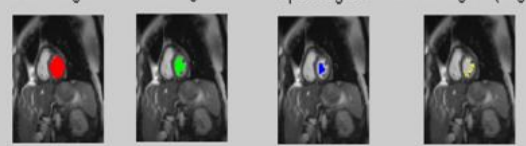

Fig.4: Automatic Segmentation using CHT with Seed point, basal (red), mid (green), Apex (blue) and Blood flow (yellow) regions.

An automated LV segmentation of MRI image starts with identifying the circular shaped province from the image. Locating the center is the chief assignment which is equipped by Round Hough Transform that stores the each cell values in the accumulator. The values more than zero stands for circle edge with high intensity and values less than zero stands for dark circles with less intensity pixel values [42]. It is observed from the LV image, the bright region which reflects radiation in the myocardium refers to blood pool and the black region which absorbs radiations refers to blockage. Two concentric circles will be marked tarnished for the inner and outer layer. Adjacent to the center, Apex point will be located, by finding the area of the inside circle. Meanwhile the area of the Apex, is very small compared to the basal and mid region area, for the measurement of blood flow volume will not be properly estimated. From the results, the area of the basal, Mid as well as Apex region has been clearly marked with different colors indicating the full depth of the left ventricle for the estimation of blood flow during the ED. In the meantime, for the same subjects manually locate the center, and estimate the area of the basal, mid and apex region. Mean, Variance and Standard deviation for all the three regions are then projected.Aimed at statistical analysis, manually calculated Mean and SD values are considered as the Ground truth values for automatic segmentation.

To find the performance measure of the proposed automatic RHT for left ventricle segmentation for both manual and auto Mean, Variance and STD are calculated for all the three regions using the Equ. (2) - Equ. (4) \& the results are tabulated in Table. 1 \& 2 From the tabulated results, the parameters Mean, variance and the $\mathrm{SD}$ of the three different regions are calculated using the Equation. (2),

$\mathrm{MEAN}=\sum \sum \frac{\mathrm{I}(\mathrm{r}, \mathrm{c})}{\mathrm{M}}$

whereI $(r, c)=$ Image Pixels \&M=Total number of pixels

VARIANCE $=\sum_{\mathrm{l}=0}^{\mathrm{M}-1}(\mathrm{I}(\mathrm{l})-\mathrm{MEAN})^{2}$

STANDARD DEVIATION $(\mathrm{SD})=\sqrt{\mathrm{VAR}}$ 
Table 1: Ground truth Segmented Mean and Standard Deviation values.

\begin{tabular}{|l|l|l|l|l|l|l|}
\hline \multirow{2}{*}{ Images } & \multicolumn{4}{l|}{ MEAN } & \multicolumn{2}{l|}{ STD } \\
\cline { 2 - 7 } & BASAL & APEX & MID & BASAL & APEX & MID \\
\hline Image 1 & 137.092 & 204.057 & 208.623 & 62.32 & 4.467 & 9.9198 \\
\hline Image 2 & 114.305 & 185.61 & 183.85 & 51.22 & 4.57 & 7.86 \\
\hline Image 3 & 129.117 & 130.331 & 129.065 & 17.73 & 8.869 & 11.404 \\
\hline Image 4 & 194.052 & 204.769 & 204.476 & 25.05 & 5.8828 & 8.516 \\
\hline
\end{tabular}

Table 2: Automatic segmented means and standard Deviation Values

\begin{tabular}{|l|c|c|c|c|c|c|}
\hline \multirow{2}{*}{ Images } & \multicolumn{3}{|c|}{ MEAX } & \multicolumn{3}{c|}{ STD } \\
\cline { 2 - 7 } & BASAL & APEX & MID & BASAL & APEX & MID \\
\hline Image 1 & 178.98 & 203.93 & 209.327 & 4.327 & 4.3449 & 9.5651 \\
\hline Image 2 & 146.082 & 185.608 & 183.834 & 45.4824 & 4.5693 & 7.8526 \\
\hline Image 3 & 123.90 & 130.48 & 129.33 & 21.657 & 9.004 & 11.5963 \\
\hline Image 4 & 179.336 & 204.838 & 202.654 & 34.734 & 5864 & 8.6491 \\
\hline
\end{tabular}

Table 3: Blood flow and Blockage in \% for automatic segmented images

\begin{tabular}{|c|c|c|}
\hline Images & $\begin{array}{c}\text { BLOOD FLOW } \\
\text { IN \% }\end{array}$ & $\begin{array}{c}\text { BLOCKAGE } \\
\text { IN \% }\end{array}$ \\
Image 1 & $\mathbf{5 8 . 2 8 9 1 2 5}$ & 41.71 \\
\hline Image 2 & 43.99461 & 56.00 \\
\hline Image 3 & 78.184961 & $\mathbf{2 1 . 8 2}$ \\
\hline Image 4 & $\mathbf{5 8 . 0 5 1 0 0 9}$ & $\mathbf{4 1 . 9 4 9}$ \\
\hline
\end{tabular}

Also for the same images $\%$ of blood flow and $\%$ of blockage of automatic method are calculated using the Equ. (5) \& Equ. (6) and tabulated in Table. 3 .

$$
\% \text { of Blood Flow }=\frac{\text { No of Pixels in the Mid Region }}{\text { No of pIxels in the Basal Region }} \times 100
$$

$\& \%$ of Blockage $=(1-$ Blood Flow $) \times 100$

To Promote Bland-Altman investigation is implemented to novelty the success of our proposed method with the existing methods. The $\mid \Delta$ Mean $\mid$ and $|\Delta \mathrm{STD}|$ for the basal, mid and apex are calculated using the Equation (6) \& (7) and tabulated for the four images in Table. 4.

$$
\begin{aligned}
|\triangle M E A N| & =\sum_{l=1}^{K} \frac{\left(M_{A N}-M_{G}-N_{A}\right)}{K} \\
M^{2} & =\text { Mean value for Ground Truth Images } \\
\text { MEAN }_{A} & =
\end{aligned}
$$

Mean value for Automatic Images (Hough Transform)

$$
\mathrm{K}=\text { Total Number of Images }
$$

$$
\begin{gathered}
\&|\Delta \mathrm{STD}|=\sum_{\mathrm{l}=1}^{\mathrm{K}} \frac{\left(\mathrm{STD}_{\mathrm{G}}-\mathrm{STD}_{\mathrm{A}}\right)}{\mathrm{K}} \\
\mathrm{STD}_{\mathrm{G}}=
\end{gathered}
$$

Standard Deviation value for Ground Truth Images

$\mathrm{STD}_{\mathrm{A}}=$ Standard Deviation value for Automatic Images

(Hough Transform)K = Total Number of Images

The Cardiac Sunny Brook Database consists of SA SSFP (Steady State Free Precession) cine image of left ventricle for ED reserved for the automatic segmentation. The end diastolic refers to largest cavity area of the cardiac cycle. Thisexperiment is implemented in MATLAB 2015 and proceeds very scarcer time to process the code with an Intel corporation core i7, 2.40 Gigahertz processor using eight GB RAM and two GB AMD Radeon graphic card on Windows 8.0. From the tabulated results fromTable.1 and Table. 2,

\begin{tabular}{|c|c|c|c|c|c|c|c|c|}
\hline \multirow{2}{*}{ Regions } & \multicolumn{2}{|c|}{ Proposed } & \multicolumn{2}{|c|}{$A R_{l}$} & \multicolumn{2}{|c|}{$A R_{:}$} & \multicolumn{2}{|c|}{$\mathbb{R}_{l} \mathbb{R}_{!}$} \\
\hline & [AIEAN] & |ASTD| & |IMEAN| & |ASTD & |MVEAN| & |ASTD| & |AMEAN| & |ASTD| \\
\hline ED BASAL (ml) & 11.932 & 2.5174 & 3.11 & 4.63 & 0.19 & 2.32 & 3.29 & 4.97 \\
\hline ED MID (ml) & 0.7035 & 0.02665 & 7.89 & 10.11 & 0.19 & 4.35 & 8.08 & 8.54 \\
\hline ED APEX (ml) & 0.28275 & 0.171 & 5.03 & 6.85 & 3.34 & 338 & 1.69 & 4.61 \\
\hline
\end{tabular}
it is observed that proposed automatic RHT segmentation Mean and STD for all the three regions found to be very adjacent to the ground truth values. Then Bland_ Altman Investigation is implemented for the Average mean of all four images for the segmented Basal, Mid and Apex for the proposed work and the existing technique results shown in Table. 4.

\section{$A R_{L}, A R_{3}, R_{1} R_{1}-$ The tabulated ralues reserved from Table 1 of $[47]$.}

The existing results AR1, $\mathbf{A} \mathbf{R}_{\mathbf{2}}, \mathbf{R}_{\mathbf{1}} \mathbf{R}_{\mathbf{2}}$ are taken from Table. 1[47]. From the investigation, it is clearly found that the proposed technique is much better than the referred existing techniques in reckoning the average Mean and Average STD for all the three regions. The accuracy of automatic segmentation is also clearly shown by the graph in Fig. 5 and Fig. 6.The graph Fig.5 clearly depicts the difference between manual and auto Mean Vs images and found that for all the three regions the difference Mean lie very close to the origin which shows that auto and manual prediction are same with good agreement. There are three outlier of basal mean indicating the overestimation by the inter-obsever erraticism. Further the graph Fig. 6 is a regression plot between automatic segmentation and manual segmentation. It concludes that the prediction of both auto and manual are almost the same as all lie on or precise close to the straight line for basal, mid and apex region.

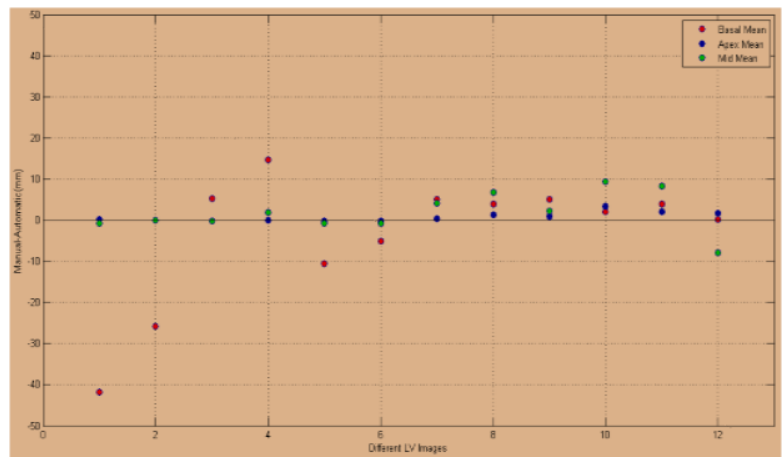

Fig.5: Difference between Manual and Auto Mean for images

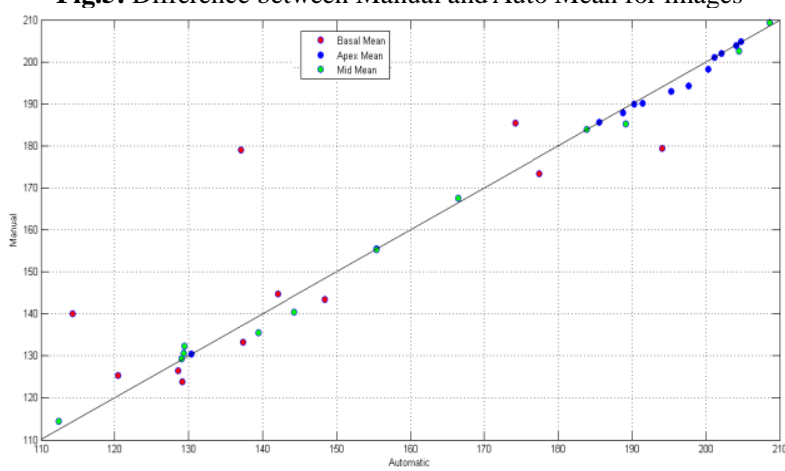

Fig.6: Linear Regression graph for Auto Vs Manual 


\section{Conclusion}

To conclude the study work, we projected a Programmed dissection of LV using RHT to locate the seed point, and segment the basal, mid and apex region of the MRI Cardiac image. The same images were used to locate the centre manually and segment the three regions. The manually estimated Mean and STD for all the three regions are considered as the ground truth values. Then compared with the automatic segmentation results and found to be very near to the ground truth values. This shows the automatic segmentation prediction is accurate to the manual observer prediction. By this segmentation, $\%$ of blood flow and \% of blockage is thencalculated and further from Bland- Altman analysis the automatic segmentation results found to be better than all the referred existing methods. The graph plot also clearly demonstrates that for all the images, the automatic RHT segmentation for all the regions found to be more close to the accuracy of ground truth values and also linear. To conclude, the automated segmentation found to be acceptable and very near to the manual ground truth segmentation and other existing automated segmentation. In future, the same automated method can be improved and implemented on video images as it will be a continuous sequential picture for all the phases of the Cardiac cycle.

\section{Acknowledgement}

Hearty thanks to the Sunnybrook Cardiac Data (SCD), Besides agreed as the 2009 Cardiac Magnetic Resonance Leftward Ventricle Dissection Experiment archives, for providing MRI left ventricle image database for research drive. The author whole heartedly thank Dr.T. Manoharan from Tamil Nadu and Dr. M. Karthikeyan from Abu Dhabi, UAE for the medicinal justification for the outcomes attained and cherished suggestions delivered to proceed the work.

\section{Conflict of retreats}

Altogether authors certainly not have any conflict of retreats to report at all times.

\section{References}

1] A.Frangi, W. Niessen, and M.A. Viergever, "ThreeDimensional Modeling for Functional Analysis of Cardiac Images: A Review", TMI, IEEE, 2001, 20(1), pp. 2-25.

[2] Lijia Wang, Mengchao Pei, Noel C.F.Codella, Minisha Kochar, Jonathan. W. Weinsaft, JianqiLi, Martin R. Prince, and Yi Wang "Left Ventricle: Fully Automated Segmentation Based on Spatiotemporal Continuity and Myocardium Information in Cine Cardiac Magnetic Resonance Imaging (LV-FAST)', HPC, BioMed Research International Vol. 2015, Article ID: 367583, pp. 1-9.

[3] K. S. L. Teo, A. Carbone, C. Piantadosi et al., "Cardiac MRI assessment of left and right ventricular parameters in healthy Australian normal Volunteers,"Heart Lung and Circulation, vol. 17 , no.4, pp.313-317, 2008

[4] L.Bonnemains, D.Mandry, P.-Y.Marie,E.Micard,B.Chen, and P.A.Vuissoz,"Assessment of right ventricle volumes and function by cardiac MRI: Quantification of the regional and global inter observer variability,"Magnetic Resonance in Medicine, vol. 67, no.6, pp.1740-1746, 2012.

[5] S. H. James, R. Wald, B. J. Wintersperger et al., "Accuracy of right and left ventricular functional assessment by short-axis vs axial cine steady-state Free-precession magnetic resonance imaging: intrapatient correlation with main pulmonary artery and ascending aorta phase-Contrast flow measurements,"Canadian Association of Radiologists Journal, vol.64, no.3, pp.213-219, 2013

[6] A.la Gerche, G. Claessen, A. van de Bruaene et al., "Cardiac MRI: a new gold standard for ventricular volume quantification during high-Intensity exercise,"Circulation: Cardiovascular Imaging, vol.6, no.2, pp.329-338, 2013.

[7] J.Caudron, J.Fares, V.Lefebvre, P.-H.Vivier, C.Petitjean, andJ.N.Dacher, "Cardiac MRI assessment of right ventricular function in acquired heart disease: factors of variability," Academic Radiology,vol.19,no.8,pp.991-1002,2012.

[8] A.Singh, L. Kurowski, and M. Y. Chiu, "Cardiac MR image segmentation using deformable models", In Proc. SPIE BMIPBMV., San Jose, USA, 1993, vol.1905, pp. 8-28.

[9] Jia Liang, Gangyi Ding, Yuwei Wu, "Segmentation of the Left Ventricle from Cardiac MR Images Based on Radial GVF Snake", DOI:10.1109/BMEI.2008.188, IEEE, pp. 238 -242, 2008

[10] T. Fujino et al., "New method of on-line quantification of regiona wall motion with automated segmental motion analysis,"J. Am. Soc.Echocardiograph, Elsevier, 2001, 14(9), pp. 892-901.

[11] J. Feng, W. Lin, C. Chen, "Epicardial boundary detection using fuzzy reasoning", TMI.,IEEE,1991,10 (2),pp.187-199.

[12] A.Lalande, M.Jaulent, "A fuzzy automaton to detect and quantify artery lesions from arteriograms", In Proc. IPMU., Granada,

[13] Spain, 1996, pp.1481-1487.

[14] H. G. Bosch, S. C. Mitchell, B. P. F. Lelieveldt, F. Nijland, O. Kamp, M. Sonka, and J. H. C. Reiber, "Active appearance-motion models for fully Automated endocardial contour detection in time sequences of echocardiograms," In Proc.CARS. Elsevier, Amsterdam, Netherlands, 2001, vol.1230, pp.941-947.

[15] T. F. Cootes, G. J. Gareth, and C. J. Taylor, "Active appearance models," TPAMI.,IEEE,2001,23(6),pp.681-685.

[16] M. Kass and A. Witkin, D. Terzo poulos, "Snake: active contour models", IJCV, Kluwer, 1988, 1(4), pp.321-331.

[17] S. Ranganth, "Contour extraction from cardiac MRI Studies using Snakes", TMI, IEEE, 1995, 14(2), pp.328-338.

[18] C. Xu, J.L. Prince, "Gradient vector flow: A new external force for snakes", In Proc.CVPR., IEEE, San Juan, Puerto Rico,1997, pp. 6671.

[19] C. Xu,J. L. Prince, "Snakes, shapes, and gradient vector flow", TIP, IEEE, 1998, pp.359-369.

[20] S. Osher, J. Sethiam, "Fronts propagating with curvature dependent speed: Algorithms based on Hamilton-Jacobi formulation,” J. Computational Phys., Elsevier, 1988, pp. 12-49.

[21] D. Mendoza, N. Codella, Y. Wang et al., "Impact of diastolic dysfunction severity on global left ventricular volumetric fillingassessment by automated segmentation of routine cine cardiovascular magnetic resonance," Journal of Cardiovascular Magnetic Resonance, vol.12, no.1, article 46, 2010.

[22] B. Grac a, P. Donato, M. J. Ferreira, M. Castelo-Branco, and F. Caseiro-Alves, "Left ventricular diastolic function in type 2 diabetes mellitus and the association with coronary artery calcium score: a cardiac MRI study," The American Journal of Roentgenology, vol.202, no.6, pp.1207-1214, 2014.

[23] N. C. F. Codella, M. D. Cham, R.Wong et al., "Rapid and accurate left ventricular chamber quantification using a novel CMR segmentation algorithm: a clinical validation study," Journal of Magnetic Resonance Imaging, vol. 31, no. 4, pp. 845-853, 2010.

[24] E. Heiberg, J. Sj ogren, M. Ugander, M. Carlsson, H. Engblom, and $\mathrm{H}$. Arheden, "Design and validation of segment-freely available software for cardiovascular image analysis," BMC Medica Imaging, vol. 10, article 1, 2010.

[25] [24] G.Messalli, A. Palumbo, E.Maffei et al., "Assessment of left ventricular volumes with cardiac MRI: comparison between two semi-automated quantitative software packages," La Radiologia Medica, vol. 114, no. 5, pp. 718-727, 2009

[26] C. Petitjean and J.-N. Dacher, "A review of segmentation methods in short axis cardiac MR images," Medical Image Analysis, vol. 15, no. 2, pp. 169-184, 2011.

[27] R. S. Inamdar andD. S. Ramdasi, "Active appearance models for segmentation of cardiac MRI data," in Proceedings of the International Conference on Communications and Signal Processing (ICCSP '13), pp. 96-100, 2013.

[28] H.Hu, H. Liu, Z. Gao, and L.Huang, "Hybrid segmentation of left ventricle in cardiac MRI using Gaussian-mixture model and region restricted dynamic programming," Magnetic Resonance Imaging, vol. 31, no. 4, pp. 575-584, 2013.

[29] Eslami, A. Karamalis, A. Katouzian, and N.Navab, "Segmentation by retrieval with guided random walks: application to left ventricle segmentation in MRI," Medical Image Analysis, vol. 17, no. 2, pp. 236-253, 2013.

[30] X. Yang, Y. Su, M. Wan et al., "Left ventricle segmentation by dynamic shape constrained random walks," in Proceedings of the 36th Annual International Conference of the IEEE Engineering in Medicine and Biology Society (EMBC '14), pp. 4723-4726, Chicago, Ill, USA, August 2014

[31] S. P. Dakua, "AnnularCut: a graph-cut design for left ventricle segmentation from magnetic resonance images," IET Image Processing, vol. 8, no. 1, pp. 1-11, 2014. 
[32] D. Grosgeorge, C. Petitjean, and S. Ruan, "Joint segmentation of right and left cardiac ventricles using multi-label graph cut," in Proceedings of the 11th IEEE International Symposium on Biomedical Imaging (ISBI '14), pp. 429-432, Beijing, China, AprilMay 2014.

[33] D. Grosgeorge, C. Petitjean, J.-N. Dacher, and S. Ruan, "Graph cut segmentation with a statistical shape model in cardiac MRI," Computer Vision and Image Understanding, vol. 117, no. 9, pp. 1027-1035, 2013.

[34] Y. Liu, C. Li, S. Guo, Y. Song, and Y. Zhao, "A novel level set method for segmentation of left and right ventricles from cardiac MR images," in Proceedings of the 36thAnnual International Conference of the IEEE Engineering in Medicine and Biology Society (EMBC '14), pp. 4719-4722, Chicago, Ill, USA, August 2014.

[35] J. A. Bessa, P. C. Cortez, J. H. da Silva F’elix, A. R. da Rocha Neto, and A. R. de Alexandria, "Radial snakes: comparison of segmentation methods in synthetic noisy images," Expert Systems with Applications, vol. 42, no. 6, pp. 3079-3088, 2015.

[36] N. C. F. Codella, J.W.Weinsaft,M. D. Cham, M. Janik, M. R. Prince, and Y.Wang, "Left ventricle: automated segmentation by using myocardial effusion threshold reduction and intra voxel computation at MR imaging," Radiology, vol. 248, no. 3, pp. 1004 1012, 2008

37] M.A.AlAttar,N. F.Osman, and A. S. Fahmy, "Segmentation of left ventricle in cardiac MRI images using adaptive multi-seeded region growing," in Proceedings of the 5th Cairo International Biomedical Engineering Conference (CIBEC '10), pp. 25-28, December 2010.

[38] S. A. Tsaftaris, V. Andermatt, A. Schlegel, A. K. Katsaggelos, D. $\mathrm{Li}$, and R. Dharma kumar, "A dynamic programming solution to tracking and elastically matching left ventricular walls in cardiac cine MRI," in Proceedings of the IEEE International Conference on Image Processing (ICIP '08), pp. 2980- 2983, San Diego, Calif, USA, October 2008

[39] A.Goshtasby and D. A.Turner, "Fusion of short-axis and long-axis cardiac MR images," Computerized Medical Imaging and Graphics, vol.20, no. 2, pp.77-87, 1996.

40] D. Saring, J. Relan, M. Groth, K. Müullerleile, and H. Handels, "Combination of short- and longaxis MR image sequences for the 3D segmentation of the left ventricle," Studies in Health Technology and Informatics, vol. 136, pp. 333-338, 2008.

[41] D. S“aring, J. Relan, M. Groth, K. M“ullerleile, and H. Handels, "3D segmentation of the left ventricle combining long- and shortaxis MR images", Methods of Information in Medicine, vol. 48, no. 4, pp. 340-343, 2009.

[42] D. Turco, C. Corsi, and C. Lamberti, "Fully automated quantification of left and right ventricular volumes throughout the cardiac cycle from magnetic resonance imaging," in Proceedings of the Computing in Cardiology (CinC '11), pp. 377-380, September 2011.

[43] John E McManigle, Richard V Stebbing, J Alison Noble, "Modified Hough Transform For Left Ventricle Myocardium Segmentation In 3-D Echocardiogram Images", BioMedIA Lab, Institute of Biomedical Engineering, Dept. of Eng. Science, University of Oxford.

[44] P. Bansod, U. B Desai, and N. Burkule, "Automatic detection of left ventricle in echocardiographic sequences using radial search andtemporalsmoothing,"in $2007,15^{\text {th }}$ InternationalConferenceonDigit alSignalProcessing, July 2007, pp. 139-142, IEEE.

45] Antonio Fernndez-Caballero and Jos M. Vega-Riesco, "Determining heart parameters through left ventricular automatic segmentation for heart disease diagnosis," Expert Syst. Appl., vol. 36, no. 2, pp, 2234-2249, Mar.2009.

[46] Shapiro, Linda and Stockman, George. "Computer Vision", Prentice-Hall, Inc. 2001

[47] T.JAtherton, D.J. Kerbyson. "Size invariant circle detection." Image and Vision Computing. Volume 17, Number 11, 1999, pp. 795-803.

[48] Uday Kurkure, Amol Pednekar, Raja Muthupillai, Scott D. Flamm, and Ioannis A. Kakadiaris, "Localization and Segmentation of Left Ventricle in Cardiac Cine-MR Images", IEEE Transactions On Biomedical Engineering, Vol. 56, No. 5, May 2009.

[49] T. Padmapriya and V. Saminadan, "Priority based fair resource allocation and Admission Control Technique for Multi-user Multiclass downlink Traffic in LTE-Advanced Networks", International Journal of Advanced Research, vol.5, no.1, pp.1633-1641, January 2017.

[50] S.V.Manikanthan and D.Sugandhi "Interference Alignment Techniques For Mimo Multicell Based On Relay Interference Broadcast Channel " International Journal of Emerging Technology in Computer Science \& Electronics (IJETCSE) ISSN: 0976-1353 Volume- 7 ,Issue 1 -MARCH 2014.
[51] Rajesh, M., and J. M. Gnanasekar. \&quot;Congestion control in heterogeneous wireless ad hoc network using FRCC.\&quot; Australian Journal of Basic and Applied Sciences 9.7 (2015): 698 702 . 